\title{
Alternative migratory tactics in brown trout (Salmo trutta) are underpinned by divergent regulation of metabolic but not neurological genes
}

\author{
Robert Wynne ${ }^{1}$, Louise Archer ${ }^{1}$, Stephen Hutton ${ }^{1}$, Luke Harman ${ }^{1}$, Patrick Gargan ${ }^{2}$, Peter \\ Moran $^{1}$, Eileen Dillane ${ }^{1}$, Jamie Coughlan ${ }^{1}$, Tom Cross $^{1}$, Philip McGinnity ${ }^{1}$, Thomas \\ Colgan $^{1}$, and Tom Reed ${ }^{1}$ \\ ${ }^{1}$ University College Cork \\ ${ }^{2}$ Inland Fisheries Ireland
}

January 19, 2021

\begin{abstract}
The occurrence of alternative morphs within populations is common but the underlying molecular mechanisms remain poorly understood. Many animals, for example, exhibit facultative migration, where two or more alternative migratory tactics (AMTs) coexist within populations. In certain salmonid species, some individuals remain in natal rivers all their lives, whilst others (in particular, females) migrate to sea for a period of marine growth. Here we performed transcriptional profiling ("RNA-seq") of the brain and liver of male and female brown trout to understand the genes and processes that differentiate migratory and residency morphs (AMT-associated genes) and how they may differ in expression between the sexes. We found tissue-specific differences with greater number of genes expressed differentially in the liver $(\mathrm{n}=867$ genes) compared to the brain $(\mathrm{n}=10)$ between the morphs. Genes with increased expression in resident livers were enriched for Gene Ontology terms associated with metabolic processes, highlighting key molecular-genetic pathways underlying the energetic requirements associated with divergent migratory tactics. In contrast, smolt-biased genes were enriched for biological processes such as response to cytokines, suggestive of possible immune function differences between smolts and residents. Finally, we identified evidence of sex-biased gene expression for AMT-associated genes in the liver $(n=18)$ but not the brain. Collectively, our results provide insights into tissue-specific gene expression underlying the production of alternative life-histories within and between the sexes, and point towards a key role for metabolic processes in the liver in mediating divergent physiological trajectories of migrants versus residents.
\end{abstract}

\section{Hosted file}

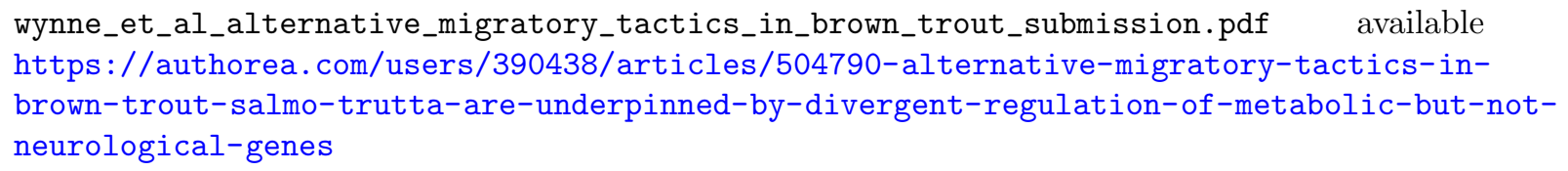



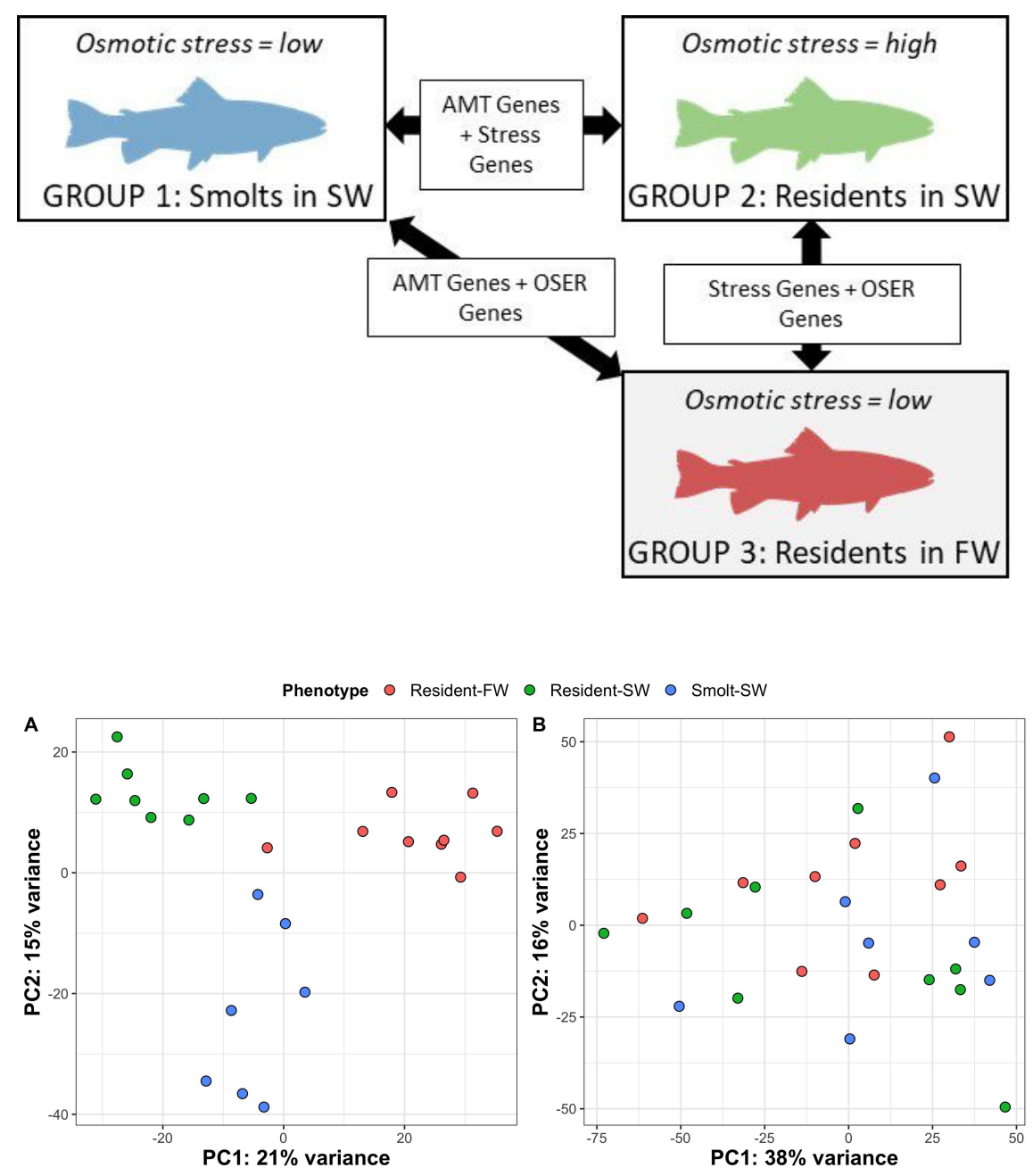

A

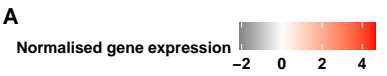

$B_{\text {Sex }} \circ \mathbf{F} \circ \mathbf{M}$

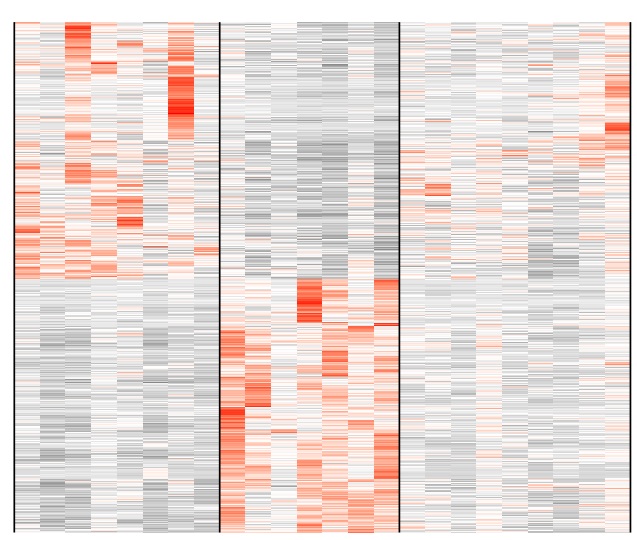

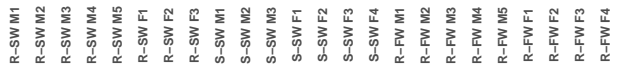

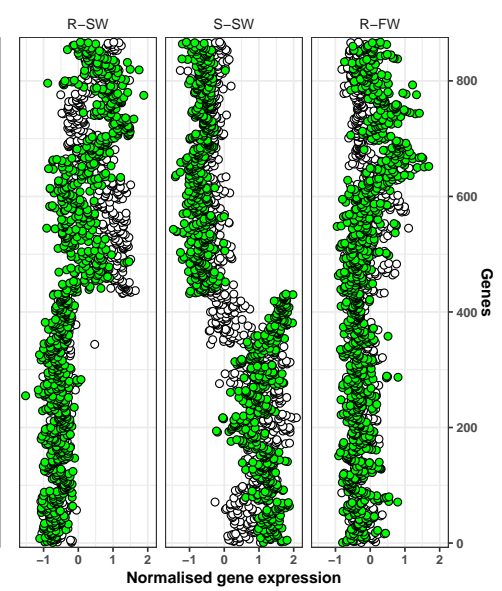

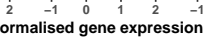




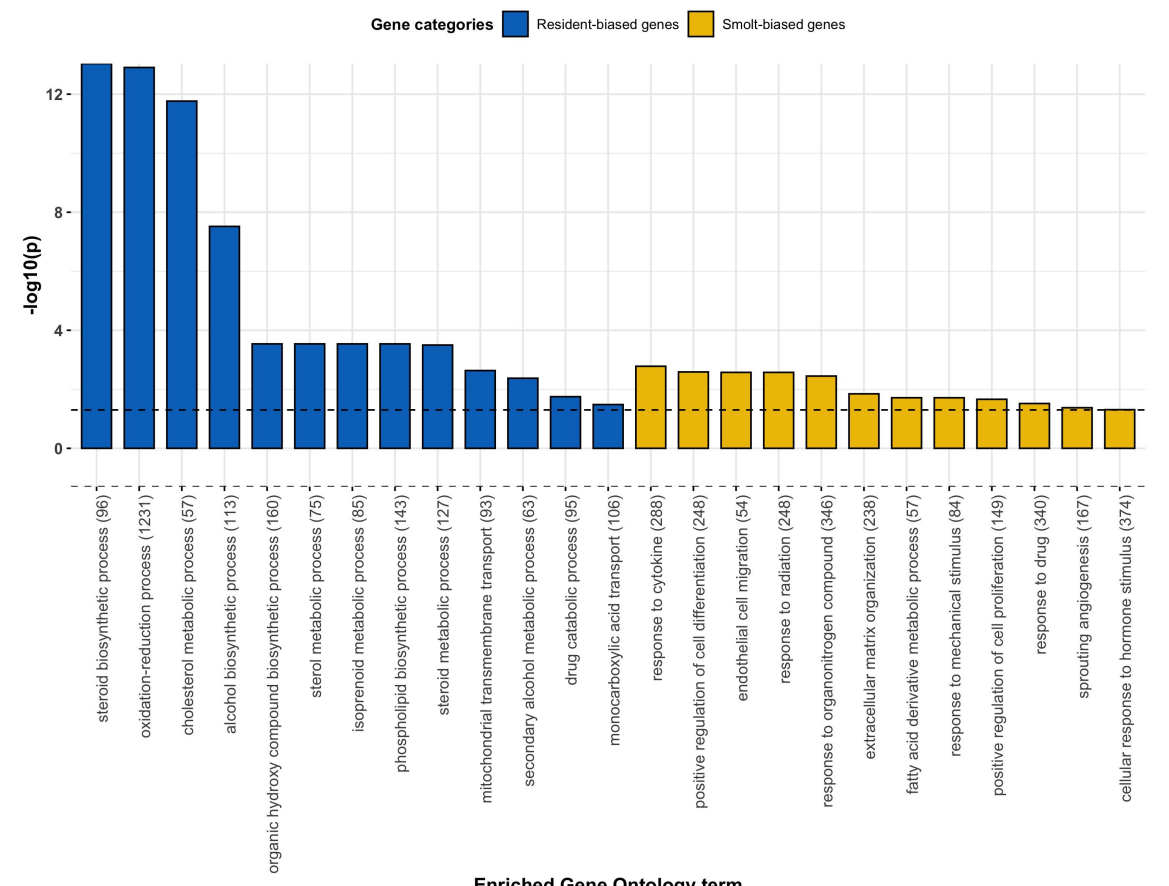

Enriched Gene Ontology term

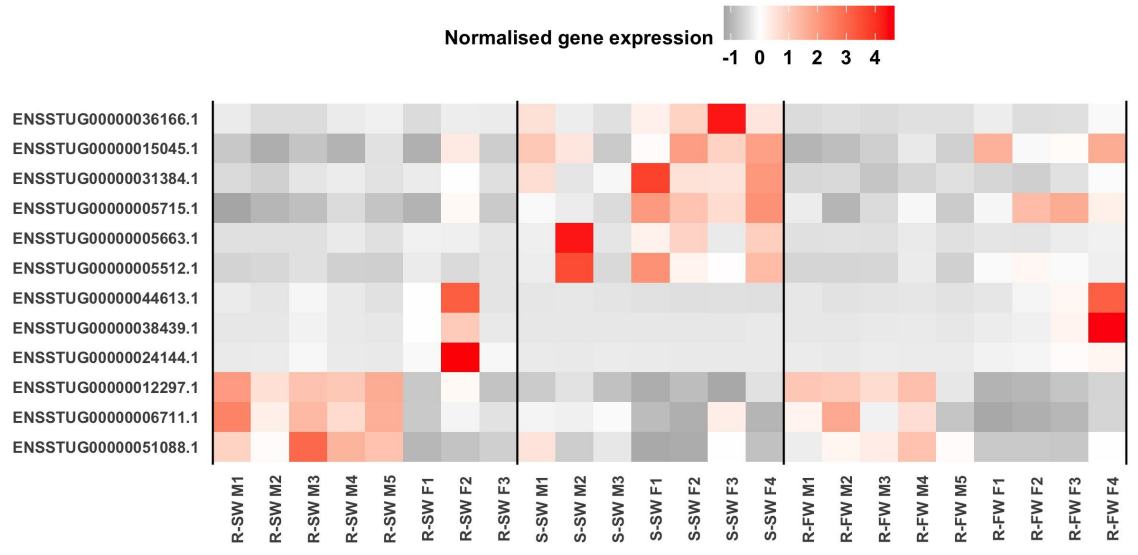

